\title{
Convergence of bounded solutions of nonlinear parabolic problems on a bounded interval: the singular case
}

\author{
Daniel Hauer
}

\begin{abstract}
In this article we prove that for every $1<p \leq 2$ and for every continuous function $f:[0,1] \times \mathbb{R} \rightarrow \mathbb{R}$, which is Lipschitz continuous in the second variable, uniformly with respect to the first one, each bounded solution of the one-dimensional heat equation

$$
u_{t}-\left\{\left|u_{x}\right|^{p-2} u_{x}\right\}_{x}+f(x, u)=0 \quad \text { in } \quad(0,1) \times(0,+\infty)
$$

with homogeneous Dirichlet boundary conditions converges as $t \rightarrow+\infty$ to a stationary solution. The proof follows an idea of Matano which is based on a comparison principle. Thus, a key step is to prove a comparison principle on non-cylindrical open sets.

Mathematics Subject Classification (1991). Primary 35B40, 35B51, 35K65.
\end{abstract}

Keywords. Asymptotic behavior, Degenerate diffusion, $p$-Laplace operator, Comparison principle.

\section{Introduction}

The aim of this paper is to study the convergence as $t \rightarrow+\infty$ of bounded solutions of the parabolic boundary value problem

$\begin{cases}u_{t}(x, t)-\left\{\left|u_{x}(x, t)\right|^{p-2} u_{x}(x, t)\right\}_{x}+f(x, u(x, t))=0 & \text { for } x \in(0,1), t>0, \\ u(0, t)=u(1, t)=0 & \text { for } t>0,\end{cases}$

to a stationary solution; i.e., a solution of the elliptic problem

$$
\left\{\begin{array}{l}
-\left\{\left|\varphi_{x}(x)\right|^{p-2} \varphi_{x}(x)\right\}_{x}+f(x, \varphi(x))=0 \quad \text { for } x \in(0,1), \\
\varphi(0)=\varphi(1)=0
\end{array}\right.
$$

This article is a part of the author's doctoral thesis and has been supported by the scholarship Mathematische Analyse von Evolution, Information und Komplexität of the Landesgraduiertenförderung Baden-Württemberg. The author wish to express his hearty gratitude to his supervisors Professor Wolfgang Arendt and Professor Ralph Chill. The author also thanks warmly Boris Andreianov for stimulating discussions. 
where $f:[0,1] \times \mathbb{R} \rightarrow \mathbb{R}$ is any continuous function such that $f(x, \cdot)$ is Lipschitz continuous on $\mathbb{R}$, uniformly for $x \in[0,1]$, and $1<p \leq 2$.

In the non-degenerate case $p=2$, Zelenyak [17] and Matano [15] have independently shown that every bounded solution of (1.1) converges to a unique stationary solution as $t \rightarrow+\infty$. The method of Zelenyak in [17] is based on a gradient inequality. It turns out that the therein used gradient inequality is in fact the so-called Eojasiewicz-Simon gradient inequality $[14,16]$, which later has been further studied and developed by several authors in order to prove convergence results and the rate of convergence of bounded solutions for many different infinite dimensional gradient or gradient-like systems (see, for example, $[10,12,4])$. The idea of Zelenyak has been revisited and extended by Haraux and Jendoubi [11, Cor. 3.2]. On the other hand, Matano's method in [15] is based on the convergence in $\mathrm{C}^{1}[0,1]$ of the solution of (1.1) to an $\omega$-limit point, a maximum principle on non-cylindrical open sets, and the unique solvability of the ordinary differential equation satisfied by stationary solutions but equipped with initial values instead of boundary values. Both methods in [17] and [15], can not directly be applied to problem (1.1) if the divergence part in (1.1) is singular (i.e. $1<p<2$ ) or degenerate (i.e., $p>2$ ) owing to the loss of regularity of the solutions and the unknown maximum principle on non-cylindrical open sets. This has already been mentioned by several authors (see $[5,6]$ ) who therein have extended the idea of Matano to obtain convergence of bounded non-negative solutions of

$$
u_{t}-\phi(u)_{x x}+g(u)_{x}+f(u)=0 \quad \text { in }(-1,1) \times(0,+\infty)
$$

with Dirichlet boundary conditions. The convergence of bounded nonnegative solutions of the equation (1.3) with Neumann or mixed boundary conditions has been studied by Gokieli and Simondon in [7].

We emphasize that the results of $[5,6]$ and [7] can not be applied to problem (1.1) due to the different character of singularity or degeneracy. Moreover, we study arbitrary solutions which are not necessarily nonnegative and we do not impose monotonicity assumptions on $f$. In particular, the set of stationary solutions can be infinite. It is not clear whether the set of stationary solutions is always discrete or can be a continuum.

The approach developed by Matano in [15] seems to be a good approach in order to establish asymptotic stability results for solutions of a large class of singular and degenerate nonlinear diffusion problems since the therein used arguments can be circumvented by regularization techniques or by using an appropriate maximum principle on non-cylindrical open sets. We note that all the above mentioned authors studying the convergence of solutions of the equation (1.3) followed closely the approach in [15]. In this article, we also follow closely the idea in [15] in all but the application of the maximum principle on non-cylindrical open sets. Since for both singular and degenerate equations such as (1.1) and (1.3) it is not clear that a maximum principle holds true, the authors in [5,6] and [7] circumvent this direct application of the maximum principle by approximating the solution $u$ of equation (1.3) by solutions 
$u_{n}$ solving a regularized equation for which the maximum principle on noncylindrical open sets is known to hold. Our idea is different to the one of $[5,6]$, and [7]: In this article, we prove a comparison principle for the equation (1.1) on non-cylindrical open sets and thus we avoid the regularization step from [5-7]. For the rest we adapt the approach used in [15] to the problem (1.1) in the singular case $1<p<2$. We outline that for every solution $u$ of problem (1.1), which is bounded with values in $L^{2}(0,1)$, the set $\{u(t) \mid t \geq 1\}$ is relatively compact in $\mathrm{C}^{1}[0,1]$ and obtain thereby that the $\omega$-limit set is contained in the set of stationary solutions, non-empty, compact, and connected in $\mathrm{C}^{1}[0,1]$. Our first main result of this article is the following.

Theorem 1.1. Let $1<p \leq 2$. Then for every global solution $u$ of (1.1), which is bounded with values in $L^{2}(0,1)$, there is a solution $\varphi$ of the stationary problem (1.2) such that

$$
u(t) \rightarrow \varphi \quad \text { in } C^{1}[0,1] \quad \text { ast } \rightarrow+\infty .
$$

This article is organized as follows. In Sect.2, we introduce a slightly more general version of problem (1.1) and we give the definition of its solutions. We show that for every solution $u$ of problem (1.1) which is bounded with values in $L^{2}(0,1)$, the set $\{u(t) \mid t \geq 1\}$ is relatively compact in $\mathrm{C}^{1}[0,1]$. At the end of Sect. 2 we state our second main theorem (Theorem 2.3). Sections 3 and 6 contain the proof of Theorem 2.3. In Sects. 4 and 5 we prove a comparison principle on non-cylindrical open sets for Dirichlet, Neumann and Robin boundary conditions, which may be of independent interest.

\section{Main result}

In fact, we study the convergence as $t \rightarrow+\infty$ of bounded solutions of the following more general problem

$$
\begin{cases}u_{t}(x, t)-a\left(x, u_{x}(x, t)\right)_{x}+f(x, u(x, t))=0 & \text { for } x \in(0,1), t>0, \\ \alpha_{0} u(0, t)-\beta_{0} a\left(0, u_{x}(0, t)\right)=0 & \text { for } t>0, \\ \alpha_{1} u(1, t)+\beta_{1} a\left(1, u_{x}(1, t)\right)=0 & \text { for } t>0\end{cases}
$$

where $f$ is given as above, $\alpha_{0}, \beta_{0}, \alpha_{1}, \beta_{1} \in[0,1]$ are such that $\alpha_{0}+\beta_{0}=1$ and $\alpha_{1}+\beta_{1}=1$, and the function $a:[0,1] \times \mathbb{R} \rightarrow \mathbb{R}$ satisfies the following suitable regularity, growth, and coercivity assumptions:

$$
\begin{cases}(x, u) \mapsto a(x, u) & \text { is continuous on }[0,1] \times \mathbb{R}, \\ u \mapsto a(x, u) & \text { is strictly increasing on } \mathbb{R}\end{cases}
$$

$\left\{\begin{array}{l}\text { there are } p \in(1,+\infty), c_{1} \in L^{p^{\prime}}(0,1), c_{2} \geq 0, \text { and } \eta>0 \text { such that } \\ |a(x, u)| \leq c_{1}(x)+c_{2}|u|^{p-1} \quad \text { for all } u \in \mathbb{R} \text { and a.e. } x \in(0,1) \\ a(x, u) u \geq \eta|u|^{p} \quad \text { for all } u \in \mathbb{R} \text { and all } x \in[0,1]\end{array}\right.$

and

$$
\left\{\begin{array}{l}
\text { there is a } b \in \mathcal{C}([0,1] \times \mathbb{R}) \text { such that } \\
b(x, \cdot) \text { is locally Lipschitz continuous, uniformly for } x \in[0,1], \\
a(x, b(x, u))=u, \quad b(x, a(x, u))=u \text { for all } u \in \mathbb{R} \text { and all } x \in[0,1]
\end{array}\right.
$$


Obviously, the prototype example of a function $a$ satisfying the hypotheses (Hi) and (Hii) is $a(x, u):=|u|^{p-1} \operatorname{sgn}(u)$ for all $(x, u) \in[0,1] \times \mathbb{R}(1<p<$ $+\infty)$. For this choice of $a$, problem (2.1) reduces to problem (1.1) from the Introduction. However, it is easily verified for this example that the inverse function $b(x, u):=|u|^{\frac{1}{p-1}} \operatorname{sgn}(u)$ is locally Lipschitz continuous if and only if $1<p \leq 2$.

It is well-known that problem $(2.1)$ can be rewritten as an abstract gradient system on $L^{2}(0,1)$ associated with the energy $\mathcal{E}: W_{0}^{1, p}(0,1) \rightarrow \mathbb{R}$ given by

$$
\mathcal{E}(u):=\int_{0}^{1} A\left(x, u_{x}(x)\right) d x+\int_{0}^{1} F(x, u(x)) d x \quad \text { for all } u \in W_{0}^{1, p}(0,1)
$$

(for Dirichlet boundary conditions), or with the energy $\mathcal{E}: W^{1, p}(0,1) \rightarrow \mathbb{R}$ given by

$$
\mathcal{E}(u):=\int_{0}^{1} A\left(x, u_{x}(x)\right) d x+\int_{0}^{1} F(x, u(x)) d x+\frac{\alpha_{0}}{2 \beta_{0}} u^{2}(0)+\frac{\alpha_{1}}{2 \beta_{1}} u^{2}(1)
$$

for all $u \in W^{1, p}(0,1)$ (for Neumann or Robin boundary conditions, $\beta_{i}>0$ ). Here $A$, and $F$ are primitives of $a$, and $f$, respectively, with respect to the second variable. Note that in order to keep this article readable we do not treat all possibilities of homogeneous mixed boundary conditions. It follows from the hypotheses $(\mathrm{Hi})$, ( $\mathrm{Hii}$, the continuity of $f$ on $[0,1] \times \mathbb{R}$, the Lipschitz continuity of $f$ in the second variable, uniformly with respect to the first one, and the theory of subdifferential operators on Hilbert spaces (see, for example, Brézis [1, Lemma 6, Proposition 7. and Proposition 8.]) that for every $u_{0} \in L^{2}(0,1)$ there exists a unique function

$$
u \in \mathrm{C}\left(\mathbb{R}_{+} ; L^{2}(0,1)\right) \cap W_{l o c}^{1, \infty}\left((0,+\infty) ; L^{2}(0,1)\right)
$$

such that

(i) the function $u$ is differentiable from the right in every $t>0$,

(ii) we have for all $t>0$,

$$
u(\cdot, t) \in W^{1, p}(0,1) \quad \text { and } \quad a\left(\cdot, u_{x}(\cdot, t)\right) \in W^{1,2}(0,1),
$$

(iii) we have

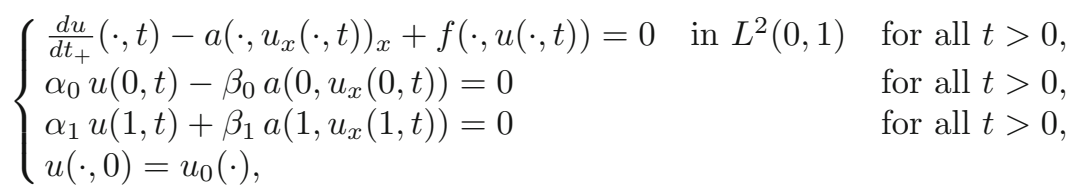

(iv) the map $t \mapsto \mathcal{E}(u(t))$ is locally absolutely continuous on $(0,+\infty)$, and

$$
\int_{t_{1}}^{t_{2}}\|\dot{u}(t)\|_{L^{2}(0,1)}^{2} d t+\mathcal{E}\left(u\left(t_{2}\right)\right)=\mathcal{E}\left(u\left(t_{1}\right)\right) \quad \text { for all } 0<t_{1}<t_{2} .
$$

We call every function $u$ satisfying the above properties a solution of (2.1). 
Proposition 2.1. Suppose that a satisfies the hypotheses (Hi) and ( $\mathrm{Hii})$. Then, every solution $u$ of problem (2.1) belongs to

$$
C\left((0,+\infty) ; C^{1}[0,1]\right) \text {. }
$$

Proof. Let $u$ be a solution of problem (2.1). We first note that by (2.5) and by the Sobolev embedding of $W^{1,2}(0,1)$ into $\mathrm{C}[0,1]$, we have $a\left(\cdot, u_{x}(\cdot, t)\right) \in \mathrm{C}[0,1]$ for all $t>0$. By hypothesis $(\mathrm{Hi})$, there is a function $b \in \mathrm{C}([0,1] \times \mathbb{R})$ such that $u_{x}(x, t)=b\left(x, a\left(x, u_{x}(x, t)\right)\right.$ for all $(x, t) \in[0,1] \times(0,+\infty)$. Thus, $u(\cdot, t) \in$ $\mathrm{C}^{1}[0,1]$ for all $t>0$. In order to prove the claim of this proposition, we show that for every $t_{0} \in(0,+\infty)$ and every sequence $\left(t_{n}\right)_{n \geq 1} \subseteq(0,+\infty)$ such that $t_{n} \rightarrow t_{0}$ as $n \rightarrow+\infty$, there exists a subsequence $\left(t_{k_{n}}\right)_{n \geq 1}$ of $\left(t_{n}\right)_{n \geq 1}$ such that

$$
u\left(\cdot, t_{k_{n}}\right) \rightarrow u\left(\cdot, t_{0}\right) \quad \text { in } \mathrm{C}^{1}[0,1] \quad \text { as } n \rightarrow+\infty,
$$

So fix $t_{0} \in(0,+\infty)$ and let $\left(t_{n}\right)_{n \geq 1} \subseteq(0,+\infty)$ be a sequence such that $t_{n} \rightarrow t_{0}$ as $n \rightarrow+\infty$. Since $u: \mathbb{R}_{+} \rightarrow L^{2}(0,1)$ is continuous and since $u \mapsto \int_{0}^{1} F(x, u(x)) d x$ maps bounded sets of $L^{2}(0,1)$ into bounded sets of $\mathbb{R}$, the sequence $\left(\int_{0}^{1} F\left(x, u\left(x, t_{n}\right)\right) d x\right)_{n \geq 1}$ is bounded. The map $t \mapsto \mathcal{E}(u(t))$ is continuous on $(0,+\infty)$ and in particular, $t \mapsto \mathcal{E}(u(t))$ is locally bounded. Hence $\left(\mathcal{E}\left(u\left(t_{n}\right)\right)\right)_{n \geq 1}$ is bounded, too. By the coercivity assumption in hypothesis (H ii), for every $t>0$,

$$
\frac{\eta}{p} \int_{0}^{1}\left|u_{x}(t)\right|^{p} d x \leq \int_{0}^{1} A\left(x, u_{x}(t)\right) d x \leq \mathcal{E}(u(t))-\int_{0}^{1} F(x, u(x, t)) d x .
$$

Hence $\left(u\left(t_{n}\right)\right)_{n \geq 1}$ is bounded in $W^{1, p}(0,1)$. By the reflexivity of $W^{1, p}(0,1)$, the compact embedding of $W^{1, p}(0,1)$ into $\mathrm{C}([0,1])$ and since $u\left(t_{n}\right) \rightarrow u\left(t_{0}\right)$ in $L^{2}(0,1)$ as $n \rightarrow+\infty$, there is a subsequence of $\left(t_{n}\right)_{n \geq 1}$, which for simplicity we denote again by $\left(t_{n}\right)_{n \geq 1}$, such that

$$
\begin{array}{ll}
u\left(t_{n}\right) \rightarrow u\left(t_{0}\right) & \text { weakly in } W^{1, p}(0,1) \text { as } n \rightarrow+\infty, \\
u\left(t_{n}\right) \rightarrow u\left(t_{0}\right) & \text { in } \mathrm{C}[0,1] \text { as } n \rightarrow+\infty .
\end{array}
$$

By the growth condition in hypothesis ( $\mathrm{Hii}),\left(a\left(\cdot, u_{x}\left(\cdot, t_{n}\right)\right)\right)_{n \geq 1}$ is bounded in $L^{p \prime}(0,1)$. We set

$$
g_{n}:=f\left(\cdot, u\left(t_{n}\right)\right)+\frac{d u}{d t_{+}}\left(t_{n}\right) \quad \text { for all } n \geq 1 .
$$

Then, by (2.11), by the continuity of $f$ and by $(2.4),\left(g_{n}\right)_{n \geq 1}$ is bounded in $L^{2}(0,1)$. Thus, and since for all $n \geq 1$, the distributional derivative $\frac{d}{d x} a\left(\cdot, u_{x}\left(\cdot, t_{n}\right)\right)=g_{n}$, we have that the sequence $\left(a\left(\cdot, u_{x}\left(\cdot, t_{n}\right)\right)\right)_{n \geq 1}$ is bounded in $W^{1,2}(0,1)$. Thus, by the compact Sobolev embedding of $W^{1,2}(0,1)$ into $\mathrm{C}[0,1]$, there is a subsequence $\left(t_{k_{n}}\right)_{n \geq 1}$ of $\left(t_{n}\right)_{n \geq 1}$ and some $\varphi \in \mathrm{C}[0,1]$ such that

$$
a\left(\cdot, u_{x}\left(\cdot, t_{k_{n}}\right)\right) \rightarrow \varphi \quad \text { in } \mathrm{C}[0,1] \quad \text { as } n \rightarrow+\infty .
$$

By hypothesis $(\mathrm{Hi})$, the map $u \mapsto b(\cdot, u)$ is continuous from $\mathrm{C}[0,1]$ to $\mathrm{C}[0,1]$ and $b(x, \cdot)$ is the inverse of $a(x, \cdot)$, for every $x \in[0,1]$. Thus

$$
u_{x}\left(\cdot, t_{k_{n}}\right) \rightarrow b(\cdot, \varphi) \quad \text { in } \mathrm{C}[0,1] \quad \text { as } n \rightarrow+\infty
$$

and so by the limit (2.10), it follows that (2.8) holds. 
Proposition 2.2. Suppose that a satisfies the hypotheses ( $\mathrm{Hi})$ and ( $\mathrm{Hii})$. If $u$ is a solution of problem (2.1) and if $u$ is bounded with values in $L^{2}(0,1)$, then for every $\delta>0$, the set $\{u(t) \mid t \geq \delta\}$ is relatively compact in $C^{1}[0,1]$.

Proof. Fix $\delta>0$. Then, obviously, it suffices to show that for every sequence $\left(t_{n}\right)_{n \geq 1} \subseteq[\delta,+\infty)$ there is a subsequence $\left(t_{k_{n}}\right)_{n \geq 1}$ of $\left(t_{n}\right)_{n \geq 1}$ such that the sequence $\left(u\left(t_{k_{n}}\right)\right)_{n \geq 1}$ converges in $\mathrm{C}^{1}[0,1]$. If $\left(t_{n}\right)_{n \geq 1}$ is bounded, then the claim is true owing to Proposition 2.1. Now, let $\left(t_{n}\right)_{n \geq 1}$ be unbounded. First, let $t, h>0$ and let $L>0$ be the Lipschitz constant of $f$ in the second variable, uniformly with respect to the first one. Then, by the monotonicity of $u \mapsto a(x, u)$ and the Lipschitz continuity of $f$,

$$
\begin{aligned}
\frac{d}{d t} \frac{1}{2}\|u(t)-u(t+h)\|_{L^{2}(0,1)}^{2}=\left(\frac{d u}{d t_{+}}(t)-\frac{d u}{d t_{+}}(t+h), u(t)-u(t+h)\right)_{L^{2}(0,1)} \\
=\left(a\left(\cdot, u_{x}(t)\right)_{x}-a\left(\cdot, u_{x}(t+h)\right)_{x}, u(t)-u(t+h)\right)_{L^{2}(0,1)} \\
\quad-(f(\cdot, u(t))-f(\cdot, u(t+h)), u(t)-u(t+h))_{L^{2}(0,1)} \\
\leq L\|u(t)-u(t+h)\|_{L^{2}(0,1)}^{2} .
\end{aligned}
$$

We integrate this inequality over $(s, t)$ for $0<s<t$. Then,

$$
\begin{aligned}
\frac{1}{2}\|u(t)-u(t+h)\|_{L^{2}(0,1)}^{2} \leq & \frac{1}{2}\|u(s)-u(s+h)\|_{L^{2}(0,1)}^{2} \\
& +L \int_{s}^{t}\|u(r)-u(r+h)\|_{L^{2}(0,1)}^{2} d r .
\end{aligned}
$$

By $(2.4), \frac{d u}{d t_{+}} \in L^{\infty}\left(s, t ; L^{2}(0,1)\right)$. Thus, dividing this inequality by $h^{2}$, letting $h \rightarrow 0+$, and using Lebesgue's dominated convergence theorem, yields to

$$
\frac{1}{2}\left\|\frac{d u}{d t_{+}}(t)\right\|_{L^{2}(0,1)}^{2} \leq \frac{1}{2}\left\|\frac{d u}{d t_{+}}(s)\right\|_{L^{2}(0,1)}^{2}+L \int_{s}^{t}\left\|\frac{d u}{d t_{+}}(r)\right\|_{L^{2}(0,1)}^{2} d r .
$$

Since the set $\{u(t) \mid t \geq 0\}$ is bounded in $L^{2}(0,1)$ and since $u \mapsto$ $\int_{0}^{1} F(x, u(x)) d x$ maps bounded sets of $L^{2}(0,1)$ into bounded sets of $\mathbb{R}$, the estimate (2.9) implies that the map $t \mapsto \mathcal{E}(u(t))$ is bounded from below. Thus, by the energy equality $(2.7), \frac{d u}{d t_{+}} \in L^{2}\left(1,+\infty ; L^{2}(0,1)\right)$ and so, by inequality (2.12),

$$
\frac{d u}{d t_{+}} \in L^{\infty}\left(1,+\infty ; L^{2}(0,1)\right) .
$$

Replacing $\left(t_{n}\right)_{n \geq 1}$ by a strictly increasing subsequence, we may assume without loss of generality that $\left(t_{n}\right)_{n \geq 1}$ is strictly increasing. Since, by hypothesis, $\left(u\left(t_{n}\right)\right)_{n \geq 1}$ is bounded in $L^{2}(0,1)$, the sequence $\left(\int_{0}^{1} F\left(x, u\left(x, t_{n}\right)\right) d x\right)_{n \geq 1}$ is bounded. By the energy equality (2.7), the map $t \mapsto \mathcal{E}(u(t))$ is non-increasing along $(0,+\infty)$ and hence, by the estimate $(2.9),\left(u\left(t_{n}\right)\right)_{n \geq 1}$ is bounded in $W^{1, p}(0,1)$. By the compact Sobolev embedding of $W^{1, p}(0,1)$ into $\mathrm{C}[0,1]$, and by the reflexivity of $W^{1, p}(0,1)$, there is a subsequence $\left(t_{k_{n}}\right)_{n \geq 1}$ of $\left(t_{n}\right)_{n \geq 1}$ and there is a $\xi \in W^{1, p}(0,1)$ such that

$$
\begin{array}{ll}
u\left(t_{k_{n}}\right) \rightarrow \xi & \text { weakly in } W^{1, p}(0,1) \quad \text { as } n \rightarrow+\infty, \text { and } \\
u\left(t_{k_{n}}\right) \rightarrow \xi & \text { in } \mathrm{C}[0,1] \quad \text { as } n \rightarrow+\infty .
\end{array}
$$


For the rest of this proof, we can now proceed analogously to the proof of Proposition 2.1.

Now, let $u$ be a solution of $(2.1)$, which is bounded with values in $L^{2}(0,1)$. Then, by Proposition 2.2, the set $\{u(t) \mid t \geq 1\}$ is relatively compact in $\mathrm{C}^{1}[0,1]$. Therefore, the $\omega$-limit set of the solution $u$ of (2.1) given by

$$
\omega(u):=\left\{\varphi \in \mathrm{C}^{1}[0,1] \mid \exists t_{n} \uparrow+\infty \text { s.t. } \lim _{n \rightarrow+\infty} u\left(t_{n}\right)=\varphi \text { in } \mathrm{C}^{1}[0,1]\right\}
$$

is nonempty and connected. Moreover, $\omega(u)$ contains exactly one element if and only if the limit $\lim _{t \rightarrow+\infty} u(t)$ exists in $\mathrm{C}^{1}[0,1]$. By the energy equality (2.7) and by LaSalle's invariance principle (see, e.g., in [9, Theorem 2.1.3 and 2.1.7]), $\omega(u)$ is contained in the set of stationary solutions of (2.1), that is, the set of solutions $\varphi$ of the elliptic problem

$$
\left\{\begin{array}{l}
\varphi \in \mathrm{C}^{1}[0,1] \text { such that } a\left(\cdot, \varphi_{x}(\cdot)\right) \in \mathrm{C}^{1}[0,1] \\
-a\left(x, \varphi_{x}(x)\right)_{x}+f(x, \varphi(x))=0 \text { for all } x \in[0,1] \\
\alpha_{0} \varphi(0)-\beta_{0} a\left(0, \varphi_{x}(0)\right)=0 \\
\alpha_{1} \varphi(1)+\beta_{1} a\left(1, \varphi_{x}(1)\right)=0 .
\end{array}\right.
$$

We are ready to formulate our main result which includes, as a special case, Theorem 1.1 .

Theorem 2.3. Assume that the hypotheses (Hi)-(Hii) are fulfilled. Then for every global solution $u$ of problem (2.1) which is bounded with values in $L^{2}(0,1)$ there is a unique solution $\varphi$ of the stationary problem (2.13) such that

$$
u(t) \rightarrow \varphi \quad \text { in } C^{1}[0,1] \quad \text { as } t \rightarrow+\infty .
$$

\section{Proof of Theorem 2.3}

Throughout this section we assume that the hypotheses $(\mathrm{Hi})-(\mathrm{Hii})$ are fulfilled and let $u$ be a global solution of problem (2.1) which is bounded with values in $L^{2}(0,1)$.

Lemma 3.1. If the $\omega$-limit set of $u$ contains more than one element, then for every $\varphi \in \omega(u)$ there is a $t_{0}>0$ such that the function $x \mapsto u\left(x, t_{0}\right)-\varphi(x)$ has at most a finite number of sign changes in $[0,1]$.

Proof. We suppose that $\omega(u)$ contains two distinct elements $\varphi$ and $\psi$. We show that there is a $\delta>0$ such that

$$
|\varphi(x)-\psi(x)|+\left|\varphi_{x}(x)-\psi_{x}(x)\right| \geq \delta \quad \text { for all } x \in[0,1] .
$$

To this end, let $\tilde{\varphi} \in \mathcal{C}^{1}[0,1]$ be a stationary solution of problem (2.1). By substituting

$$
\nu_{1}=\tilde{\varphi} \quad \text { and } \quad \nu_{2}=a\left(\cdot, \tilde{\varphi}_{x}\right),
$$

the differential equation

$$
-a\left(x, \tilde{\varphi}_{x}(x)\right)_{x}+f(x, \tilde{\varphi}(x))=0
$$


is equivalent to the two-dimensional first order system

$$
\nu_{1}^{\prime}=b\left(\cdot, \nu_{2}\right), \quad \nu_{2}^{\prime}=f\left(\cdot, \nu_{1}\right) .
$$

Due to the hypotheses, both maps $b$ and $f$ are locally Lipschitz continuous in the second variable, uniformly with respect to the first one. Thus, for every $x_{0} \in[0,1], \nu_{0,1}, \nu_{0,2} \in \mathbb{R}$, the first order system (3.2) with initial values

$$
\nu_{1}\left(x_{0}\right)=\nu_{0,1}, \nu_{2}\left(x_{0}\right)=\nu_{0,2}
$$

has a unique solution. Therefore, if we assume for two different $\varphi, \psi \in \omega(u)$ that there is an $x_{0} \in[0,1]$ such that

$$
\left|\varphi\left(x_{0}\right)-\psi\left(x_{0}\right)\right|+\left|\varphi_{x}\left(x_{0}\right)-\psi_{x}\left(x_{0}\right)\right|=0,
$$

then the above stated local uniqueness of the initial value problem associated with the first order system (3.2) implies that $\varphi=\psi$. But this obviously contradicts to our hypothesis that $\varphi$ and $\psi$ are distinct elements of $\omega(u)$.

Since $\psi \in \omega(u)$, there is sequence $\left(t_{n}\right)_{n \geq 1} \subseteq(0,+\infty)$ such that $t_{n} \uparrow+\infty$ and $u\left(t_{n}\right) \rightarrow \psi$ in $\mathrm{C}^{1}[0,1]$ as $n \rightarrow+\infty$. Hence, and by using the inequality (3.1), there is a $t_{0}>0$ such that

$$
\left|u\left(x, t_{0}\right)-\varphi(x)\right|+\left|u_{x}\left(x, t_{0}\right)-\varphi_{x}(x)\right| \geq \frac{\delta}{2} \quad \text { for all } x \in[0,1] .
$$

Now, we suppose that the function $x \mapsto u\left(x, t_{0}\right)-\varphi(x)$ admits infinitely many sign changes in the interval $[0,1]$ and we shall reach a contradiction. In fact, by the intermediate value theorem, there is a sequence $\left(x_{n}\right)_{n \geq 1} \subseteq[0,1]$ of pairwise different zeros. We can extract a monotone subsequence $\left(x_{k_{n}}\right)_{n \geq 1} \subseteq$ $\left(x_{n}\right)_{n \geq 1}$ and we find a zero $x_{0} \in[0,1]$ of the function $u\left(\cdot, t_{0}\right)-\varphi(\cdot)$ such that $x_{k_{n}} \rightarrow x_{0}$ as $n \rightarrow+\infty$. By Rolle's theorem, there is a second sequence $\left(\xi_{k_{n}}\right)_{n \geq 1} \subseteq[0,1]$ of pairwise different zeros of the derivative $u_{x}\left(\cdot, t_{0}\right)-\varphi_{x}(\cdot)$ such that $x_{k_{n}}<\xi_{k_{n}}<x_{k_{n+1}}$ (respectively, $x_{k_{n}}>\xi_{k_{n}}>x_{k_{n+1}}$ ) for every $n \geq 1$. Thus $\xi_{k_{n}} \rightarrow x_{0}$ as $n \rightarrow+\infty$, too, and it turns out that the point $x_{0}$ is also a zero of the derivative $u_{x}\left(\cdot, t_{0}\right)-\varphi_{x}(\cdot)$ in $[0,1]$. But this contradicts to inequality (3.3).

\section{A comparison principle for Dirichlet boundary conditions}

The following notation is similar to the one used by Brown et al. in [3]. For given $\left(x_{0}, t_{0}\right) \in \mathbb{R}^{2}$ and $\rho>0$ we denote by $Q\left(\left(x_{0}, t_{0}\right), \rho\right)$ the cylinder

$$
\left\{(x, t) \in \mathbb{R}^{2}|| x-x_{0} \mid<\rho, t_{0}-\rho<t<t_{0}\right\} .
$$

Let $\mathcal{C}$ be an open subset of $\mathbb{R}^{2}$. If $\partial \mathcal{C}$ denotes its topological boundary and $\mathcal{C}^{c}$ the complement of $\mathcal{C}$ with respect to $\mathbb{R}^{2}$, then we define by

$$
\mathcal{P C}:=\left\{(x, t) \in \partial \mathcal{C} \mid Q((x, t), \rho) \cap \mathcal{C}^{c} \neq \emptyset \text { for all } \rho>0\right\}
$$

the parabolic boundary of $\mathcal{C}$. We note that if the set $\mathcal{C}$ is a bounded cylinder, i.e., $\mathcal{C}=\left(a_{0}, b_{0}\right) \times\left(t_{0}, t_{1}\right)$, then

$$
\mathcal{P C}=\left(\left\{a_{0}, b_{0}\right\} \times\left[t_{0}, t_{1}\right]\right) \cup\left(\left[a_{0}, b_{0}\right] \times\left\{t_{0}\right\}\right) .
$$


For the following, let $a: \mathcal{C} \times \mathbb{R} \rightarrow \mathbb{R}$ satisfy

$$
\left\{\begin{array}{l}
(x, t) \mapsto a(x, t, u) \quad \text { is measurable on } \mathcal{C} \text { for all } u \in \mathbb{R}, \\
u \mapsto a(x, t, u) \quad \text { is continuous and increasing for every }(x, t) \in \mathcal{C}, \\
\text { there are } p \in(1,+\infty), c_{1} \in L^{p \prime}(\mathcal{C}), \text { and } c_{2} \geq 0 \text { such that } \\
|a(x, t, u)| \leq c_{1}(x, t)+c_{2}|u|^{p-1} \quad \text { for all } u \in \mathbb{R} \text { and a.e. }(x, t) \in \mathcal{C},
\end{array}\right.
$$

and let $f: \mathcal{C} \times \mathbb{R} \rightarrow \mathbb{R}$ be measurable in the first two variables, Lipschitz continuous in the third variable with constant $L>0$, uniformly with respect to the first two, and $f(\cdot, \cdot, 0) \in L^{2}(\mathcal{C})$.

For a continuous function $u: \overline{\mathcal{C}} \rightarrow \mathbb{R}$, we denote by $u^{+}:=\max \{0, u\}$ its positive part and for every $T \in \mathbb{R}$, we define $\mathcal{C}_{T}:=\{(x, t) \in \mathcal{C} \mid t<T\}$. If the set $\mathcal{C}$ is bounded from below, then we denote by $t_{\text {bot }}$ the infimum of all $t \in \mathbb{R}$ for which there exists an $x \in \mathbb{R}$ such that $(x, t) \in \mathcal{C}$.

Definition 4.1. Let $\mathcal{C} \subseteq \mathbb{R}^{2}$ be an open subset. We say that two continuous functions $u$ and $v$ on $\overline{\mathcal{C}}$ satisfy the inequality

$$
\begin{aligned}
& u_{t}(x, t)-a\left(x, t, u_{x}(x, t)\right)_{x}+f(x, t, u(x, t)) \\
& \quad \leq v_{t}(x, t)-a\left(x, t, v_{x}(x, t)\right)_{x}+f(x, t, v(x, t))
\end{aligned}
$$

on $\mathcal{C}$ if for all bounded sub-cylinders $Q=\left(a_{0}, b_{0}\right) \times\left(t_{0}, t_{1}\right) \subseteq \mathcal{C}$,

$$
u, v \in W^{1,2}\left(t_{0}, t_{1} ; L^{2}\left(a_{0}, b_{0}\right)\right) \cap \mathrm{C}\left(\left[t_{0}, t_{1}\right] ; W^{1, p}\left(a_{0}, b_{0}\right)\right)
$$

and for all non-negative $\xi \in \mathrm{C}_{c}^{1}(\mathcal{C})$,

$$
\begin{aligned}
\int_{C}\left[u_{t}\right. & \left.-v_{t}\right] \xi d(x, t)+\int_{C}\left[a\left(x, t, u_{x}\right)-a\left(x, t, v_{x}\right)\right] \xi_{x} d(x, t) \\
& +\int_{C}[f(x, t, u)-f(x, t, v)] \xi d(x, t) \leq 0 .
\end{aligned}
$$

Lemma 4.2. (Comparison principle for Dirichlet boundary conditions) Let $\mathcal{C} \subseteq$ $\mathbb{R}^{2}$ be an open subset such that for all $T \in \mathbb{R}, \mathcal{C}_{T}$ is bounded and topological regular, that is, the interior int $\left(\overline{\mathcal{C}_{T}}\right)=\mathcal{C}_{T}$. If $u$ and $v$ are two continuous functions on $\overline{\mathcal{C}}$ satisfying the inequality (4.1) on $\mathcal{C}$, then

$$
\sup _{(x, t) \in \overline{\mathcal{C}}} e^{-L\left(t-t_{\text {bot }}\right)}(u-v)(x, t) \leq \sup _{(x, t) \in \mathcal{P C}} e^{-L\left(t-t_{\text {bot }}\right)}[u-v]^{+}(x, t) .
$$

Remark 4.3. We note that for Lemma 4.2, we have been inspired by a comparison principle of a linear unilateral problem without Lipschitz-perturbation given by Brézis in [2, Proposition II.5]. See also the maximum principle for classical solutions of the linear inhomogeneous heat equation in Ladyženskaja et al. [13, Theorem 2.1]. We point out that Lemma 4.2 remains true if $f: \mathcal{C} \times \mathbb{R} \rightarrow \mathbb{R}$ is only locally Lipschitz continuous in the third variable, uniformly with respect to the first two, and if $u$ and $v$ are uniformly bounded. In this case, the constant $L$ in (4.3) depends on bounds of $u$ and $v$.

Proof. Part I: First we consider the case when $\mathcal{C}$ is the bounded cylinder $\left(a_{0}, b_{0}\right) \times\left(t_{0}, t_{1}\right)$. Obviously, then $t_{0}=t_{b o t}$. Let $u$ and $v$ satisfy the inequality $(4.1)$ on $\mathcal{C}$. We set

$$
k:=\sup _{(x, t) \in \mathcal{P C}} e^{-L\left(t-t_{0}\right)}[u-v]^{+}(x, t) \text { and } w(x, t):=u(x, t)-v(x, t)-k e^{L\left(t-t_{0}\right)}
$$


for all $(x, t) \in \overline{\mathcal{C}}$. Then

$$
w^{+} \in W^{1,2}\left(t_{0}, t_{1} ; L^{2}\left(a_{0}, b_{0}\right)\right) \cap \mathcal{C}\left(\left[t_{0}, t_{1}\right] ; W^{1, p}\left(a_{0}, b_{0}\right)\right)
$$

with the weak derivatives

$$
\frac{d}{d t} w^{+}(x, t)=\left[u_{t}(x, t)-v_{t}(x, t)-k L e^{L\left(t-t_{0}\right)}\right] \cdot \mathbb{1}_{\left\{u-v>k e^{L\left(t-t_{0}\right)}\right\}}
$$

and

$$
\frac{d}{d x} w^{+}(x, t)=\left[u_{x}(x, t)-v_{x}(x, t)\right] \cdot \mathbb{1}_{\left\{u-v>k e^{L\left(t-t_{0}\right)}\right\}}
$$

for a.e. $(x, t) \in \mathcal{C}$. Here we denote by $\mathbb{1}_{\left\{u-v>k e^{L\left(t-t_{0}\right)}\right\}}$ the characteristic function of the set

$$
\left\{u-v>k e^{L\left(t-t_{0}\right)}\right\}:=\left\{(x, t) \in \mathcal{C} \mid u(x, t)-v(x, t)>k e^{L\left(t-t_{0}\right)}\right\} .
$$

Moreover, $w^{+}(x, t)=0$ for all $(x, t) \in \mathcal{P C}$. Recall that for every $t \in\left(t_{0}, t_{1}\right), \mathcal{C}_{t}$ denotes the cylinder $\left(a_{0}, b_{0}\right) \times\left(t_{0}, t\right)$. Note that for cylinders $\mathcal{C}$, an approximation argument shows that inequality (4.2) remains true for non-negative function

$$
\xi \in W^{1,2}\left(t_{0}, t_{1} ; L^{2}\left(a_{0}, b_{0}\right)\right) \cap \mathcal{C}\left(\left[t_{0}, t_{1}\right] ; W^{1, p}\left(a_{0}, b_{0}\right)\right)
$$

such that $\xi=0$ on $\mathcal{P C}$. Thus, by the monotonicity of $u \mapsto a(x, u)$ (hypotheses $(\mathrm{Hi})$ ), and by the Lipschitz continuity of $f$, we obtain that for all $t \in\left(t_{0}, t_{1}\right)$,

$$
\begin{aligned}
& \frac{1}{2} \int_{a_{0}}^{b_{0}} w^{+}(x, t)^{2} d x=\int_{a_{0}}^{b_{0}} \int_{t_{0}}^{t} \frac{d}{d s} \frac{1}{2}\left(w^{+}(x, s)\right)^{2} d s d x \\
&=\int_{\mathcal{C}_{t}} \frac{d}{d s} w^{+}(x, s) w^{+}(x, s) d(x, s) \\
&=\int_{\mathcal{C}_{t}}\left[u_{s}(x, s)-v_{s}(x, s)\right] w^{+}(x, s) d(x, s)-\int_{\mathcal{C}_{t}} k L e^{L\left(s-t_{0}\right)} w^{+}(x, s) d(x, s) \\
& \leq-\int_{\mathcal{C}_{t}}\left[a\left(x, s, u_{x}(x, s)\right)-a\left(x, s, v_{x}(x, s)\right)\right] \\
& \quad \times\left[u_{x}(x, s)-v_{x}(x, s)\right] \mathbb{1}_{\left\{u-v>k e^{L\left(s-t_{0}\right)}\right\}} d(x, s) \\
& \quad-\int_{\mathcal{C}_{t}}[f(x, s, u(x, s))-f(x, s, v(x, s))] w^{+}(x, s) d(x, s) \\
& \quad-\int_{\mathcal{C}_{t}} k L e^{L\left(s-t_{0}\right)} w^{+}(x, s) d(x, s) \\
& \leq L \int_{\mathcal{C}_{t}}[u(x, s)-v(x, s)] w^{+}(x, s) d(x, s)-\int_{\mathcal{C}_{t}} k L e^{L\left(s-t_{0}\right)} w^{+}(x, s) d(x, s) \\
&= L \int_{t_{0}}^{t} \int_{a_{0}}^{b_{0}} w^{+}(x, s)^{2} d x d s .
\end{aligned}
$$

Thus, by Gronwall's inequality (see Gronwall [8]) and by the continuity of $w$ on $\overline{\mathcal{C}}$, we deduce from the above estimates that $w^{+}(x, t)=0$ for every $(x, t) \in \overline{\mathcal{C}}$. This shows that the inequality (4.3) holds when $\mathcal{C}$ is a cylinder.

Part II: Now, we assume that the open nonempty set $\mathcal{C} \subseteq \mathbb{R}^{2}$ is as in the assumption. Let $u$ and $v$ satisfy the inequality (4.1) on $\mathcal{C}$ and let $T>t_{\text {bot }}$. 
We prove the inequality (4.3) for $\mathcal{C}=\mathcal{C}_{T}$ and for unbounded $\mathcal{C}$ let $T$ tend to $+\infty$. We set

$$
k:=\sup _{(x, t) \in \mathcal{P C}} e^{-L\left(t-t_{b o t}\right)}[u-v]^{+}(x, t),
$$

and

$$
w(x, t):=u(x, t)-v(x, t)-k e^{L\left(t-t_{\text {bot }}\right)}, \quad \text { for all }(x, t) \in \overline{\mathcal{C}} .
$$

Let $\varepsilon>0$. Since the function $w^{+}$is uniformly continuous on $\overline{\mathcal{C}}$ and $w^{+}=0$ on $\mathcal{P C}$, there exists a $\delta>0$ such that

$$
w^{+}(x, t)<\varepsilon \quad \text { for all }(x, t) \in \Sigma_{\delta}:=\{(x, t) \in \overline{\mathcal{C}} \mid d((x, t), \mathcal{P C}) \leq \delta\} .
$$

Here we denote by $d((x, t), A):=\inf _{(y, s) \in A} \max \{|x-y|,|t-s|\}$ the distance of a bounded set $A \subseteq \mathbb{R} \times(0,+\infty)$ and the point $(x, t)$ with respect to the supremum norm. Furthermore, we set

$$
\mathcal{C}_{\delta}:=\{(x, t) \in \overline{\mathcal{C}} \mid d((x, t), \mathcal{P C})>\delta / 2\}
$$

1st step: We set

$$
t_{\text {top }}:=\sup \{t \in \mathbb{R} \mid \exists x \in \mathbb{R} \text { s.t. }(x, t) \in \mathcal{C}\} .
$$

Since the set $\mathcal{C}$ is bounded, open and nonempty, $-\infty<t_{\text {bot }}<t_{\text {top }}<+\infty$. We may always assume that $t_{\text {top }}-t_{\text {bot }}>\delta$; otherwise we choose $\delta>0$ smaller. We choose an integer $n_{0} \geq 3$ such that $\frac{t_{t o p}-t_{\text {bot }}-\delta}{n_{0}} \leq \frac{\delta}{2}$ and set

$$
t_{0}:=t_{b o t}+\frac{\delta}{2}, \quad t_{1}:=t_{0}+\frac{t_{t o p}-t_{b o t}-\delta}{n_{0}} .
$$

Since the points in $\overline{\mathcal{C}_{\delta}} \subseteq \overline{\mathcal{C}}$ have positive distance to the parabolic boundary, and by definition of the parabolic boundary,

for every $(x, t) \in \overline{\mathcal{C}_{\delta}}$ there exists an $\rho>0$ such that $Q((x, t), \rho) \subseteq \mathcal{C}$.

As a consequence, there exists an open set $\ell_{1} \subseteq \mathbb{R}$ such that

$$
\mathcal{C}_{\delta} \cap\left\{t=t_{1}\right\}=\ell_{1} \times\left\{t_{1}\right\} .
$$

Since $\ell_{1}$ is open, there is a family $\left(\left(a_{i}^{(1)}, b_{i}^{(1)}\right)\right)_{i \in I_{1}}$ of open and pairwise disjoint intervals such that $\ell_{1}=\bigcup_{i \in I_{1}}\left(a_{i}^{(1)}, b_{i}^{(1)}\right)$. Let $Q_{i}^{(1)}:=\left(a_{i}^{(1)}, b_{i}^{(1)}\right) \times\left(t_{0}, t_{1}\right)$. We first show that $Q_{i}^{(1)} \subseteq \mathcal{C}$. By (4.4) and a compactness argument, there exists a $\rho>0$ such that $\left(a_{i}^{(1)}, b_{i}^{(1)}\right) \times\left(t_{1}-\rho, t_{1}\right) \subseteq \mathcal{C}$. Hence, if we define

$$
t^{\prime}:=\inf \left\{t \in\left(t_{0}, t_{1}\right) \mid\left(a_{i}^{(1)}, b_{i}^{(1)}\right) \times\left(t, t_{1}\right) \subseteq \mathcal{C}\right\}
$$

then $t_{0} \leq t^{\prime}<t_{1}$ and

$$
Q_{1}:=\left(a_{i}^{(1)}, b_{i}^{(1)}\right) \times\left(t^{\prime}, t_{1}\right) \subseteq \mathcal{C} .
$$

Assume that $t^{\prime}>t_{0}$. Note that for every $x \in\left[a_{i}^{(1)}, b_{i}^{(1)}\right]$, one has $\left(x, t^{\prime}\right) \in$ $\overline{Q_{i}^{(1)}} \subseteq \overline{\mathcal{C}}$ and

$$
d\left(\left(x, t^{\prime}\right), \mathcal{P C}\right) \geq d\left(\left(x, t_{1}\right), \mathcal{P C}\right)-\left(t_{1}-t^{\prime}\right) \geq \delta / 2-\left(t_{1}-t^{\prime}\right)>0,
$$


so that $\left[a_{i}^{(1)}, b_{i}^{(1)}\right] \times\left\{t^{\prime}\right\} \subseteq \overline{\mathcal{C}}$ has no intersection with the parabolic boundary. A compactness argument again implies that there exists a $\rho^{\prime}>0$ such that

$$
Q_{2}:=\left(a_{i}^{(1)}, b_{i}^{(1)}\right) \times\left(t^{\prime}-\rho^{\prime}, t^{\prime}\right) \subseteq \mathcal{C} .
$$

As a consequence,

$$
\left(a_{i}^{(1)}, b_{i}^{(1)}\right) \times\left(t^{\prime}-\rho^{\prime}, t_{1}\right)=\operatorname{int}\left(\overline{Q_{1} \cup Q_{2}}\right) \subseteq \operatorname{int}(\overline{\mathcal{C}})=\mathcal{C} .
$$

But this is a contradiction to the definition of $t^{\prime}$ if $t^{\prime}>t_{0}$. Hence, $Q_{i}^{(1)} \subseteq \mathcal{C}$. It follows from (4.4) and the definition of $\mathcal{C}_{\delta}$ that

$$
d\left(\left(a_{i}^{(1)}, t_{1}\right), \mathcal{P C}\right) \leq \delta / 2 \quad \text { and } \quad d\left(\left(b_{i}^{(1)}, t_{1}\right), \mathcal{P C}\right) \leq \delta / 2 .
$$

By the triangle inequality, for every $t \in\left[t_{0}, t_{1}\right]$,

$$
d\left(\left(a_{i}^{(1)}, t\right), \mathcal{P C}\right) \leq d\left(\left(a_{i}^{(1)}, t_{1}\right), \mathcal{P C}\right)+\left(t_{1}-t\right) \leq \delta / 2+\delta / 2=\delta
$$

and similarly, $d\left(\left(b_{i}^{(1)}, t\right), \mathcal{P C}\right) \leq \delta$ so that $\left\{a_{i}^{(1)}, b_{i}^{(1)}\right\} \times\left[t_{0}, t_{1}\right] \subseteq \Sigma_{\delta}$. Moreover, by the definition of $t_{0},\left[a_{i}^{(1)}, b_{i}^{(1)}\right] \times\left\{t_{0}\right\} \subseteq \Sigma_{\delta}$, so that all together $\mathcal{P} Q_{i}^{(1)} \subseteq \Sigma_{\delta}$.

Since for $\tilde{u}:=u-k e^{L\left(t-t_{\text {bot }}\right)}$,

$$
(f(x, \tilde{u})-f(x, u))-k L e^{L\left(t-t_{b o t}\right)} \leq 0,
$$

we find that $\tilde{u}$ and $v$ verify on $\mathcal{C}$ that

$$
\begin{aligned}
\tilde{u}_{t}-a\left(x, \tilde{u}_{x}\right)_{x}+f(x, \tilde{u})= & u_{t}-k L e^{L\left(t-t_{b o t}\right)}-a\left(x, u_{x}\right)_{x}+f(x, \tilde{u}) \\
= & u_{t}-a\left(x, u_{x}\right)_{x}+f(x, u)+(f(x, \tilde{u})-f(x, u)) \\
& -k L e^{L\left(t-t_{b o t}\right)} \leq v_{t}-a\left(x, v_{x}\right)_{x}+f(x, v) .
\end{aligned}
$$

Thus, it follows by inequality (4.3) applied to $\tilde{u}$ and $v$ on the cylinder $Q_{i}^{(1)}$ (proved in part I) that

$$
w^{+}(x, t) \leq e^{L \cdot \frac{t_{t o p}-t_{b o t}-\delta}{n_{0}}} \varepsilon
$$

for all $(x, t) \in \overline{Q_{i}^{(1)}}$. Since this estimate is independent of the index $i$, we have that $w^{+}$satisfies $(4.5)$ in $\bigcup_{i \in I_{1}} \overline{Q_{i}^{(1)}}$.

2nd Step: Let

$$
t_{2}:=t_{b o t}+\frac{\delta}{2}+2 \frac{t_{t o p}-t_{b o t}-\delta}{n_{0}} .
$$

There exists again a family $\left(\left(a_{i}^{(2)}, b_{i}^{(2)}\right)\right)_{i \in I_{2}}$ of open and pairwise disjoint intervals $\left(a_{i}^{(2)}, b_{i}^{(2)}\right)$ such that

$$
\mathcal{C}_{\delta} \cap\left\{t=t_{2}\right\}=\bigcup_{i \in I_{2}}\left(a_{i}^{(2)}, b_{i}^{(2)}\right) \times\left\{t_{2}\right\} .
$$

Let $Q_{i}^{(2)}=\left(a_{i}^{(2)}, b_{i}^{(2)}\right) \times\left(t_{1}, t_{2}\right)$. Similarly as before, one shows that $Q_{i}^{(2)} \subseteq \mathcal{C}$. Moreover, the lateral boundary $\left\{a_{i}^{(2)}, b_{i}^{(2)}\right\} \times\left[t_{1}, t_{2}\right] \subseteq \Sigma_{\delta}$, and the bottom $\left[a_{i}^{(2)}, b_{i}^{(2)}\right] \times\left\{t_{1}\right\}$ is contained in $\left(\ell_{1} \times\left\{t_{1}\right\}\right) \cup \Sigma_{\delta}$. Thus, we have that for every $i \in I_{2}, w^{+}$satisfies the estimate (4.5) on the parabolic boundary $\mathcal{P} Q_{i}^{(2)}$ and so

$$
w^{+}(x, t) \leq e^{L \cdot 2 \cdot \frac{t_{t o p}-t_{b o t}-\delta}{n_{0}}} \varepsilon
$$


for all $(x, t) \in \bigcup_{i \in I_{2}} \overline{Q_{i}^{(2)}} \cup \bigcup_{i \in I_{1}} \overline{Q_{i}^{(1)}}$. Now, for every $3 \leq n \leq n_{0}$, let

$$
t_{n}:=t_{b o t}+\frac{\delta}{2}+n \frac{t_{t o p}-t_{b o t}-\delta}{n_{0}} \text {. }
$$

We find again a family $\left(\left(a_{i}^{(n)}, b_{i}^{(n)}\right)\right)_{i \in I_{n}}$ of open and pairwise disjoint intervals $\left(a_{i}^{(n)}, b_{i}^{(n)}\right)$ such that

$$
\mathcal{C}_{\delta} \cap\left\{t=t_{n}\right\}=\bigcup_{i \in I_{n}}\left(a_{i}^{(2)}, b_{i}^{(2)}\right) \times\left\{t_{2}\right\} .
$$

We set

$$
\ell_{n}:=\bigcup_{i \in I_{n}}\left(a_{i}^{(n)}, b_{i}^{(n)}\right) \quad \text { and } \quad Q_{i}^{(n)}=\left(a_{i}^{(n)}, b_{i}^{(n)}\right) \times\left(t_{n-1}, t_{n}\right) .
$$

By the same reasoning as given for $n=2$, we obtain that the cylinder $Q_{i}^{(n)} \subseteq \mathcal{C}$, the lateral boundary $\left\{a_{i}^{(n)}, b_{i}^{(n)}\right\} \times\left[t_{n-1}, t_{n}\right] \subseteq \Sigma_{\delta}$, and the bottom $\left[a_{i}^{(n)}, b_{i}^{(n)}\right] \times$ $\left\{t_{n-1}\right\}$ is contained in $\ell_{n-1} \cup \Sigma_{\delta}$. Thus for each $3 \leq n \leq n_{0}$, we obtain that

$$
w^{+}(x, t) \leq e^{L \cdot n \cdot \frac{t_{t o p}-t_{\text {bot }}-\delta}{n_{0}}} \varepsilon \quad \text { for every }(x, t) \in \bigcup_{i=1}^{n} \bigcup_{j \in I_{i}} \overline{Q_{j}^{(i)}}
$$

and in particular for $n=n_{0}$, we have that

$$
\begin{gathered}
w^{+}(x, t) \leq e^{L\left(t_{\text {top }}-t_{\text {bot }}-\delta\right)} \varepsilon \leq e^{L\left(t_{\text {top }}-t_{\text {bot }}\right)} \varepsilon \\
\text { for all }(x, t) \in \mathcal{Q}_{\delta, n_{0}}:=\bigcup_{i=1}^{n_{0}} \bigcup_{j \in I_{i}} Q_{j}^{(i)} .
\end{gathered}
$$

Since $\mathcal{C}_{\delta} \subseteq \mathcal{Q}_{\delta, n_{0}}$, this estimate in particular holds for all $(x, t) \in \mathcal{C}_{\delta}$. By construction,

$$
w^{+}(x, t) \leq \varepsilon \leq e^{L\left(t_{t o p}-t_{b o t}\right)} \varepsilon \quad \text { for all }(x, t) \in \Sigma_{\delta}
$$

and $\mathcal{C}_{\delta} \cup \Sigma_{\delta}=\mathcal{C}$. Thus

$$
w^{+}(x, t) \leq e^{L\left(t_{t o p}-t_{b o t}\right)} \varepsilon \quad \text { for all }(x, t) \in \mathcal{C}
$$

and so by letting $\varepsilon \rightarrow 0+$ in this inequality, the claim of this lemma holds.

\section{A comparison principle for Neumann or Robin boundary conditions}

For the sequel we need the following notation. If $\mathcal{C}$ is an open subset of $[0,1] \times \mathbb{R}$, then we denote by

$$
\mathcal{S}_{0} \mathcal{C}:=\{(0, t) \in \mathcal{P C} \mid \exists \rho>0 \text { s.t. } Q((\rho, t+\rho), \rho) \subseteq \overline{\mathcal{C}}\}
$$

the left lateral boundary of $\mathcal{C}$ touching the vertical line $\{0\} \times \mathbb{R}$, and by

$$
\mathcal{S}_{1} \mathcal{C}:=\{(1, t) \in \mathcal{P C} \mid \exists \rho>0 \text { s.t. } Q((1-\rho, t+\rho), \rho) \subseteq \overline{\mathcal{C}}\}
$$

the right lateral boundary of $\mathcal{C}$ touching the vertical line $\{1\} \times \mathbb{R}$. 
We suppose that $f$ satisfies the same hypotheses as for the Dirichlet boundary conditions and that $a$ satisfies

$$
\left\{\begin{array}{l}
(x, t) \mapsto a(x, t, u) \quad \text { is continuous on } \mathcal{C} \text { for all } u \in \mathbb{R} \\
u \mapsto a(x, t, u) \quad \text { is continuous and increasing for every }(x, t) \in \mathcal{C}, \\
\text { there are } p \in(1,+\infty), c_{1} \in L^{p \prime}(\mathcal{C}), \text { and } c_{2} \geq 0 \text { such that } \\
|a(x, t, u)| \leq c_{1}(x, t)+c_{2}|u|^{p-1} \quad \text { for all } u \in \mathbb{R} \text { and a.e. }(x, t) \in \mathcal{C} .
\end{array}\right.
$$

Definition 5.1. Let $\mathcal{C} \subseteq[0,1] \times \mathbb{R}$ be an open subset, $\alpha_{0}, \alpha_{1} \in[0,1), I_{0}, I_{1} \subseteq \mathbb{R}$ be two open subsets such that $\{0\} \times I_{0} \subseteq \mathcal{S}_{0} \mathcal{C}$ and $\{1\} \times I_{1} \subseteq \mathcal{S}_{1} \mathcal{C}$, and let $\varphi_{0}, \psi_{0} \in \mathrm{C}\left(I_{0}\right)$ and $\varphi_{1}, \psi_{1} \in \mathrm{C}\left(I_{1}\right)$. We say that two continuous functions $u$ and $v$ on $\overline{\mathcal{C}}$ satisfy the inequality (4.1) together with the Neumann (if $\alpha_{0}=\alpha_{1}=0$ ) or Robin boundary conditions

$$
\begin{cases}\alpha_{0} u(0, t)-a\left(0, t, u_{x}(0, t)\right)=\varphi_{0}(t) & \text { for all } t \in I_{0}, \\ \alpha_{1} u(1, t)+a\left(1, t, u_{x}(1, t)\right)=\varphi_{1}(t) & \text { for all } t \in I_{1}\end{cases}
$$

and

$$
\begin{cases}\alpha_{0} v(0, t)-a\left(0, t, v_{x}(0, t)\right)=\psi_{0}(t) & \text { for all } t \in I_{0} \\ \alpha_{1} v(1, t)+a\left(1, t, v_{x}(1, t)\right)=\psi_{1}(t) & \text { for all } t \in I_{1}\end{cases}
$$

if for every bounded sub-cylinder $Q=\left(a_{0}, b_{0}\right) \times\left(t_{0}, t_{1}\right) \subseteq \mathcal{C}$,

$$
u, v \in W^{1,2}\left(t_{0}, t_{1} ; L^{2}\left(a_{0}, b_{0}\right)\right) \cap \mathrm{C}\left(\left[t_{0}, t_{1}\right] ; W^{1, p}\left(a_{0}, b_{0}\right)\right)
$$

and for all non-negative $\xi \in \mathrm{C}_{c}^{1}(\overline{\mathcal{C}})$ with $\xi=0$ on $\mathcal{P C} \backslash\left(\left(\{0\} \times I_{0}\right) \cup\left(\{1\} \times I_{1}\right)\right)$, the inequality

$$
\begin{aligned}
\int_{\mathcal{C}}\left(u_{t}-v_{t}\right) \xi d(x, t)+\int_{\mathcal{C}}\left[a\left(x, t, u_{x}\right)-a\left(x, t, v_{x}\right)\right] \xi_{x} d(x, t) \\
-\left\{\int_{I_{1}}\left[\varphi_{1}(t)-\psi_{1}(t)+\alpha_{1}(v(1, t)-u(1, t))\right] \xi(1, t) d t\right. \\
\left.-\int_{I_{0}}\left[\alpha_{0}(u(0, t)-v(0, t))+\left(\psi_{0}(t)-\varphi_{0}(t)\right)\right] \xi(0, t) d t\right\} \\
+\int_{\mathcal{C}}[f(x, t, u)-f(x, t, v)] \xi d(x, t) \leq 0
\end{aligned}
$$

holds.

Lemma 5.2. (Comparison principle for Neumann or Robin boundary conditions) Let $\mathcal{C}$ be an open subset of $[0,1] \times \mathbb{R}$ such that for all $T \in \mathbb{R}, \mathcal{C}_{T}$ is bounded and topological regular. Let $L \geq 0$ be the Lipschitz-constant of $f, \alpha_{0}, \alpha_{1} \in[0,1), I_{0}, I_{1} \subseteq \mathbb{R}$ be open subsets such that $\{0\} \times I_{0} \subseteq \mathcal{S}_{0} \mathcal{C},\{1\} \times I_{1} \subseteq$ $\mathcal{S}_{1} \mathcal{C}, \varphi_{0}, \psi_{0} \in C\left(I_{0}\right)$, and $\varphi_{1}, \psi_{1} \in C\left(I_{1}\right)$. If $u$ and $v$ are two continuous function on $\overline{\mathcal{C}}$ satisfying the inequality (4.1) on $\mathcal{C}$ together with Neumann or Robin boundary conditions (5.1) and (5.2) and if

$$
\left\{\begin{array}{l}
\varphi_{0}(t) \leq \psi_{0}(t) \quad \text { for all } t \in I_{0} \\
\varphi_{1}(t) \leq \psi_{1}(t) \quad \text { for all } t \in I_{1}
\end{array}\right.
$$


then

$$
\begin{aligned}
& \sup _{(x, t) \in \overline{\mathcal{C}}} e^{-L\left(t-t_{\text {bot }}\right)}(u-v)(x, t) \\
& \quad \leq \sup _{(x, t) \in \mathcal{P} \mathcal{C} \backslash\left(\left(\{0\} \times I_{0}\right) \cup\left(\{1\} \times I_{1}\right)\right)} e^{-L\left(t-t_{\text {bot }}\right)}[u-v]^{+}(x, t) .
\end{aligned}
$$

Proof. Part I: First, we show that the inequality (5.5) holds when $\mathcal{C}$ is the bounded cylinder $\left(a_{0}, b_{0}\right) \times\left(t_{0}, t_{1}\right), I_{0}$ and $I_{1}$ are open subsets of $\left(t_{0}, t_{1}\right)$, and $u$ and $v$ satisfy the inequality (4.1) on $\mathcal{C}$ in connection with the boundary conditions (5.1) on $\left\{a_{0}\right\} \times I_{0}$ and (5.2) on $\left\{b_{0}\right\} \times I_{1}$. We set

$$
k:=\sup _{(x, t) \in \mathcal{P C} \backslash\left(\left(\{0\} \times I_{0}\right) \cup\left(\{1\} \times I_{1}\right)\right)} e^{-L\left(t-t_{0}\right)}[u-v]^{+}(x, t)
$$

and

$$
w(x, t):=u(x, t)-v(x, t)-k e^{L\left(t-t_{0}\right)}
$$

for all $(x, t) \in \overline{\mathcal{C}}$. Then $w^{+} \in W^{1,2}\left(t_{0}, t_{1} ; L^{2}\left(a_{0}, b_{0}\right)\right) \cap \mathcal{C}\left(\left[t_{1}, t_{2}\right] ; W^{1, p}\left(a_{0}, b_{0}\right)\right)$ and $w^{+}=0$ on $\mathcal{P C} \backslash\left(\left(\left\{a_{0}\right\} \times I_{0}\right) \cup\left(\left\{b_{0}\right\} \times I_{1}\right)\right)$. By the condition (5.4) on $\left.\left(\left\{a_{0}\right\} \times I_{0}\right) \cup\left(\left\{b_{0}\right\} \times I_{1}\right)\right)$, we have that

$$
\begin{aligned}
& \left\{\int_{\left\{b_{0}\right\} \times I_{1}}\left[\varphi_{1}(t)-\psi_{1}(t)+\alpha_{1}\left(v\left(b_{0}, t\right)-u\left(b_{0}, t\right)\right)\right] w^{+}\left(b_{0}, t\right) d t\right. \\
& \left.\quad-\int_{\left\{a_{0}\right\} \times I_{0}}\left[\alpha_{0}\left(u\left(a_{0}, t\right)-v\left(a_{0}, t\right)\right)+\left(\psi_{0}(t)-\varphi_{0}(t)\right)\right] w^{+}\left(a_{0}, t\right) d t\right\} \leq 0 .
\end{aligned}
$$

Thus, we can use the same arguments as in the part I of the proof of Lemma 4.2 when we replace the therein used inequality (4.2) by the inequality (5.3).

Part II: Now, let $\mathcal{C} \subseteq[0,1] \times \mathbb{R}$ be a general open and subset as stated in the hypothesis of this lemma. We fix $T>t_{\text {bot }}$ and we first assume that $\mathcal{C}=\mathcal{C}_{T}$. We set

$$
k:=\sup _{(x, t) \in \mathcal{P C} \backslash\left(\left(\{0\} \times I_{0}\right) \cup\left(\{1\} \times I_{1}\right)\right)} e^{-L\left(t-t_{0}\right)}[u-v]^{+}(x, t)
$$

and

$$
w(x, t):=u(x, t)-v(x, t)-k e^{L\left(t-t_{b o t}\right)} \quad \text { for all }(x, t) \in \overline{\mathcal{C} \cap\{t<T\}} .
$$

Let $\varepsilon>0$. Then, by the uniform continuity of $w^{+}$on $\overline{\mathcal{C}}$ and since

$$
w^{+}=0 \quad \text { on } \quad \mathcal{P C} \backslash\left(\left(\{0\} \times I_{0}\right) \cup\left(\{1\} \times I_{1}\right)\right),
$$

there is a $\delta>0$ such that

$$
w^{+}(x, t)<\varepsilon \quad \text { for all }(x, t) \in \Sigma_{\delta(\varepsilon)}
$$

where

$$
\Sigma_{\delta(\varepsilon)}:=\left\{(x, t) \in \overline{\mathcal{C}} \mid d\left((x, t), \mathcal{P C} \backslash\left(\left(\{0\} \times I_{0}\right) \cup\left(\{1\} \times I_{1}\right)\right)\right) \leq \delta\right\} .
$$

Next, we set

$$
\mathcal{C}_{\delta}:=\left\{(x, t) \in \overline{\mathcal{C}} \mid d\left((x, t), \mathcal{P C} \backslash\left(\left(\{0\} \times I_{0}\right) \cup\left(\{1\} \times I_{1}\right)\right)\right)>\delta / 2\right\} .
$$


Now, we can proceed analogously to the part II of the proof of Lemma 4.2 where for the estimates on the cylinders $Q_{i}^{(j)}$ we use inequality (5.5) (proved in part I) instead of inequality (4.3).

\section{Proof of Theorem 2.3 (Continued)}

We define for every $\varphi \in \omega(u)$ the sets

$$
A^{+}(\varphi):=\{(x, t) \in[0,1] \times(0,+\infty) \mid u(x, t)-\varphi(x)>0\}
$$

and

$$
A^{-}(\varphi):=\{(x, t) \in[0,1] \times(0,+\infty) \mid u(x, t)-\varphi(x)<0\} .
$$

Furthermore, we call a connected component $\mathcal{C}$ of $A^{+}(\varphi)$ (resp., of $A^{-}(\varphi)$ ) a maximal open and connected subset of $A^{+}(\varphi)$ (resp., of $A^{-}(\varphi)$ ).

Lemma 6.1. Let $\varphi \in \omega(u)$ and let $t_{0}>0$. Then for every nonempty connected component $\mathcal{C}$ of the set

$$
A^{+}(\varphi) \cap\left([0,1] \times\left[t_{0},+\infty\right)\right)
$$

there is an $x_{0} \in[0,1]$ such that $\left(t_{0}, x_{0}\right) \in \mathcal{C}$. The same assertion also holds for the set

$$
A^{-}(\varphi) \cap\left([0,1] \times\left[t_{0},+\infty\right)\right) .
$$

Proof. We only prove the first claim since the second claim is shown similarly. To do so, we assume there is a nonempty connected component $\mathcal{C}$ of the set (6.1) and there is no element $x \in[0,1]$ such that $\left(x, t_{0}\right) \in \mathcal{C}$. We replace $\mathcal{C}$ by its topological regularization $\operatorname{int}(\overline{\mathcal{C}})$. Let $S_{0}:=\{0\} \times(0,+\infty)$ and $S_{1}:=\{1\} \times(0,+\infty)$. Since $u=\varphi$ on $\mathcal{P C} \backslash\left(S_{0} \cup S_{1}\right)$ and since $u$ and $\varphi$ satisfy on $\mathcal{P C} \cap\left(S_{0} \cup S_{1}\right)$, either again $u=\varphi$, or the inequalities (5.4) for the boundary conditions (5.1) and (5.2), we obtain by the comparison principle with respect to the appropriate boundary conditions (Lemma 4.2, or Lemma 5.2, respectively) that $u=\varphi$ on $\overline{\mathcal{C}}$. But this contradicts that $\mathcal{C}$ is a subset of $A^{+}(\varphi)$ and therefore the claim of this lemma holds true.

Lemma 6.2. Suppose the $\omega$-limit set $\omega(u)$ contains more than one element. Then, for every $\varphi \in \omega(u)$, there exists a $t_{0}>0$ such that the functions $t \mapsto$ $u(0, t)-\varphi(0)$ and $t \mapsto u_{x}(0, t)-\varphi_{x}(0)$ do not change the sign along $\left[t_{0},+\infty\right)$.

Proof. We take $\varphi \in \omega(u)$ and chose $t_{0}>0$ such that the function $u\left(\cdot, t_{0}\right)-\varphi(\cdot)$ has only a finite number of sign changes in $[0,1]$, according to Lemma 3.1 .

First, we show that the function $t \mapsto u(0, t)-\varphi(0)$ has only a finite number of sign changes on $\left[t_{0},+\infty\right)$. If $\beta_{0}=0$, then there is nothing to show. So we assume that $\beta_{0}>0$. We suppose that the function $u(0, \cdot)-\varphi(0)$ has infinitely many sign changes along $\left[t_{0},+\infty\right)$. Then there is a strictly increasing sequence $\left(t_{n}\right)_{n \geq 1}$ such that for all $n \geq 1, u\left(0, t_{2 n-1}\right)-\varphi(0)>0$ and $u\left(0, t_{2 n}\right)-\varphi(0)<0$, that is, for all $n \geq 1$,

$$
\begin{aligned}
\left(0, t_{2 n-1}\right) & \in A^{+}(\varphi) \cap\left([0,1] \times\left[t_{0},+\infty\right)\right) \quad \text { and } \\
\left(0, t_{2 n}\right) & \in A^{-}(\varphi) \cap\left([0,1] \times\left[t_{0},+\infty\right)\right) .
\end{aligned}
$$


Let for each $n \geq 1$,

$\mathcal{C}_{2 n-1} \subseteq A^{+}(\varphi) \cap\left([0,1] \times\left[t_{0},+\infty\right)\right) \quad$ and $\quad \mathcal{C}_{2 n} \subseteq A^{-}(\varphi) \cap\left([0,1] \times\left[t_{0},+\infty\right)\right)$ denote the connected component such that $\left(0, t_{n}\right) \in \mathcal{C}_{n}$. Then, due to Lemma 6.1, there is a continuous curve $\gamma_{n} \subseteq \mathcal{C}_{n}$ and there is a point $x_{n} \in[0,1]$ such that $\gamma_{n}$ connects $\left(0, t_{n}\right)$ with $\left(x_{n}, t_{0}\right)$. Since for all $n \geq 1, \gamma_{2 n-1}$ is contained in $A^{+}(\varphi)$ and $\gamma_{2 n}$ in $A^{-}(\varphi)$, the sequence $\left(x_{n}\right)_{n \geq 1}$ is strictly increasing and by construction, $u\left(x_{2 n-1}, t_{0}\right)-\varphi\left(x_{2 n-1}\right)>0$ and $u\left(x_{2 n}, t_{0}\right)-\varphi\left(x_{2 n}\right)<0$ for all $n \geq 1$. But this obviously contradicts to our choice of $t_{0}$ and hence the first claim of this lemma holds.

Next, we show that the function $t \mapsto u_{x}(0, t)-\varphi_{x}(0)$ has only a finite number of sign changes on $\left[t_{0},+\infty\right)$. Here, due to the monotonicity of the function $u \mapsto a(x, u)$, it obviously suffices to treat the case when $\beta_{0}<1$. We take $\varphi \in \omega(u)$, choose $t_{0}>0$ as given by Lemma 3.1, and assume again there is a strictly increasing sequence $\left(t_{n}\right)_{n \geq 1} \subseteq\left(t_{0},+\infty\right)$ such that for every $n \geq 1$,

$$
u_{x}\left(0, t_{2 n-1}\right)-\varphi_{x}(0)>0 \quad \text { and } \quad u_{x}\left(0, t_{2 n}\right)-\varphi_{x}(0)<0 .
$$

If $\beta_{0}=0$, then for all $n \geq 1, u\left(0, t_{n}\right)-\varphi(0)=0$. Since $u\left(\cdot, t_{n}\right) \in \mathrm{C}^{1}[0,1]$, we find a sequence $\left(x_{n}\right)_{n \geq 1}$ of points $x_{n} \in(0,1)$ such that in view of $(6.2)$, for every $n \geq 1$, the segments

$$
\left(0, x_{2 n-1}\right] \times\left\{t_{2 n-1}\right\} \subseteq A^{+}(\varphi) \cap\left([0,1] \times\left[t_{0},+\infty\right)\right)
$$

and

$$
\left(0, x_{2 n}\right] \times\left\{t_{2 n}\right\} \subseteq A^{-}(\varphi) \cap\left([0,1] \times\left[t_{0},+\infty\right)\right) .
$$

If $\beta_{0} \in(0,1)$, then by the monotonicity of $a(x, \cdot)$ and in view to $(6.2)$, for all $n \geq 1$,

$$
\begin{aligned}
u\left(0, t_{2 n-1}\right)-\varphi(0) & =\frac{\beta_{0}}{\alpha_{0}}\left(a\left(0, u_{x}\left(0, t_{2 n-1}\right)\right)-a\left(0, \varphi_{x}(0)\right)\right)>0 \quad \text { and } \\
u\left(0, t_{2 n}\right)-\varphi(0) & <0 .
\end{aligned}
$$

By the continuity of $u\left(\cdot, t_{n}\right)-\varphi(\cdot)$ on $[0,1]$ for each $n \geq 1$, we find again a sequence $\left(x_{n}\right)_{n \geq 1} \subseteq(0,1)$ such that for all $n \geq 1,(6.3)$ and (6.4) hold.

Now, for $\beta_{0} \in[0,1)$ and for every $n \geq 1$, let $\mathcal{C}_{n}$ be the connected component such that

$$
\left(x_{2 n-1}, t_{2 n-1}\right) \in \mathcal{C}_{2 n-1} \subseteq A^{+}(\varphi) \cap\left([0,1] \times\left[t_{0},+\infty\right)\right)
$$

and

$$
\left(x_{2 n}, t_{2 n}\right) \in \mathcal{C}_{2 n} \subseteq A^{-}(\varphi) \cap\left([0,1] \times\left[t_{0},+\infty\right)\right) .
$$

Then by Lemma 6.1 , for every $n \geq 1$, there is a point $y_{n} \in[0,1]$ such that $\left(y_{n}, t_{0}\right) \in \mathcal{C}_{n}$ and so we find a continuous path $\gamma_{n}$ in $\mathcal{C}_{n}$ connecting $\left(x_{n}, t_{n}\right)$ and $\left(y_{n}, t_{0}\right)$. Now, for every $n \geq 1$, we denote by $\kappa_{n}$ the path from $\left(0, t_{n}\right)$ to $\left(y_{n}, t_{0}\right)$ by running along both paths $\left[0, x_{n}\right] \times\left\{t_{n}\right\}$ and $\gamma_{n}$. Due to this construction we have that the path $\kappa_{2 n-1} \backslash\left\{\left(0, t_{2 n-1}\right)\right\} \subseteq A^{+}(\varphi)$ and $\kappa_{2 n} \backslash\left\{\left(0, t_{2 n}\right)\right\} \subseteq A^{-}(\varphi)$ for every $n \geq 1$. Thus the curves $\left(\kappa_{n}\right)_{n}$ cannot intersect each other and hence the sequence $\left(y_{n}\right)_{n \geq 1}$ is strictly increasing and for all $n \geq 1, u\left(y_{2 n-1}, t_{0}\right)-$ $\varphi\left(y_{2 n-1}\right)>0$ and $u\left(y_{2 n}, t_{0}\right)-\varphi\left(y_{2 n}\right)<0$ But this is again a contradiction to the choice of $t_{0}>0$. 
Proof of Theorem 2.3 We assume that the set $\omega(u)$ is not discrete. Since $\omega(u)$ is non-empty and connected, it then contains infinitely many elements. We take three distinct elements of $\omega(u)$ and denote them $\varphi^{1}, \varphi^{2}$, and $\varphi^{3}$. Because of the different boundary conditions in the stationary problem (2.13), we need to treat two cases:

The case $\alpha_{0}=1$ : Owing to the hypotheses $(\mathrm{Hi})$, and ( $\left.\mathrm{Hii}\right)$, the initial value problem

$$
\left\{\begin{array}{l}
-a\left(x, v_{x}(x)\right)_{x}+f(x, v(x))=0 \quad \text { for } x \in[0,1], \\
v(0)=0, \quad v_{x}(0)=v_{0}
\end{array}\right.
$$

admits for every $v_{0} \in \mathbb{R}$ a unique solution $v$ on $[0,1]$. Thus, we may assume without loss of generality that

$$
\varphi_{x}^{1}(0)<\varphi_{x}^{2}(0)<\varphi_{x}^{3}(0) .
$$

According to Lemma 6.2, there is a $t_{0}>0$ such that the function $t \mapsto u_{x}(0, t)-$ $\varphi_{x}^{2}(0)$ has constant sign along the interval $\left[t_{0},+\infty\right)$. First, we assume that $u_{x}(0, t) \geq \varphi_{x}^{2}(0)$ for every $t \geq t_{0}$. Since $\varphi^{1} \in \omega(u)$, there is an unbounded strictly increasing sequence $\left(t_{n}\right)_{n} \subseteq(0,+\infty)$ such that

$$
u_{x}\left(t_{n}\right) \rightarrow \varphi_{x}^{1} \quad \text { in } \mathcal{C}[0,1] \text { as } n \rightarrow+\infty .
$$

But on the other hand, due to inequality (6.5), we have

$$
0<\varphi_{x}^{2}(0)-\varphi_{x}^{1}(0) \leq u_{x}(0, t)-\varphi_{x}^{1}(0) \leq\left\|u_{x}(t)-\varphi_{x}^{1}\right\|_{\mathcal{C}[0,1]} \quad \text { for all } t \geq t_{0},
$$

which contradicts to the convergence in (6.6) and hence $\varphi^{1}$ can not be an element of $\omega(u)$. Similarly, we obtain that $\varphi^{3}$ can not belong to $\omega(u)$ if $u_{x}(0, t) \leq$ $\varphi_{x}^{2}(0)$ for all $t \geq t_{0}$. Thus, in this case the set $\omega(u)$ contains at most two different elements, which contradicts to our assumption that $\omega(u)$ is not discrete. The case $\alpha_{0} \in[0,1)$ : Since under the hypotheses (Hi), and ( $\left.\mathrm{Hii}\right)$, the initial value problem

$$
\left\{\begin{array}{l}
-a\left(x, v_{x}(x)\right)_{x}+f(x, v(x))=0 \\
v(0)=v_{0}, \quad a\left(0, v_{x}(0)\right)=\frac{\alpha_{0}}{\beta_{0}} v(0)
\end{array} \text { for } x \in[0,1],\right.
$$

possesses for every $v_{0} \in \mathbb{R}$ a unique solution $v$ on $[0,1]$, the values $\varphi^{1}(0), \varphi^{2}(0)$, and $\varphi^{3}(0)$ have to be different from one another. Now, arguing as in the case $\alpha_{0}=1$ yields that also in this case the set $\omega(u)$ contains at most two different elements and therefore also in this case our assumption is false. This shows that the claim of Theorem 2.3 holds.

\section{References}

[1] Brézis, H.: Propriétés régularisantes de certains semi-groupes non linéaires. Israel J. Math. 9, 513-534 (1971)

[2] Brézis, H.: Problèmes unilatéraux, J. Math. Pures Appl. 9(51), 1-168 (1972)

[3] Brown, R.M., Hu, W., Lieberman, G.M.: Weak solutions of parabolic equations in non-cylindrical domains. Proc. Am. Math. Soc. 125(6), 1785-1792 (1997) 
[4] Chill, R.: On the Łojasiewicz-Simon gradient inequality. J. Funct. Anal. 201(2), $572-601(2003)$

[5] Falliero, M., Simondon, F.: Convergence pour un problème parabolique dégénéré: une remarque, Publ. Math. UFR Sci. Tech. Besancon, Univ. Franche-Comté 17 (2003)

[6] Feireisl, E., Simondon, F.: Convergence for degenerate parabolic equations. J. Differ. Equ. 152(2), 439-466 (1999)

[7] Gokieli, M., Simondon, F.: Convergence to equilibrium for a parabolic problem with mixed boundary conditions in one space dimension. J. Evol. Equ. 3(04), 523-548 (2004)

[8] Gronwall, T.H.: Note on the derivatives with respect to a parameter of the solutions of a system of differential equations. Ann. Math. 20(4), 292-296 (1919)

[9] Haraux, A.: Systèmes dynamiques dissipatifs et applications, Masson (1991)

[10] Haraux, A., Jendoubi, M.A.: Decay estimates to equilibrium for some evolution equations with an analytic nonlinearity. Asymptot. Anal. 26(1), 21-36 (2001)

[11] Haraux, H., Jendoubi, M.A.: On the convergence of global and bounded solutions of some evolution equations, J. Evol. Equ. 7, 449-470 (2007)

[12] Haraux, A., Jendoubi, M.A., Kavian, O.: Rate of decay to equilibrium in some semilinear parabolic equations. Dedicated to Philippe Bénilan. J. Evol. Equ. 3(3), 463-484 (2003)

[13] Ladyženskaja, O.A., Solonnikov, V.A., Ural'ceva, N.N.: Linear and Quasilinear Equations of Parabolic Type, American Mathematical Society (1986) (Translations of Mathematical Monographs)

[14] Łojasiewicz, S.: Une propriété topologique des sous-ensembles analytiques réels, Colloques internationaux du C.N.R.S.: Les équations aux dérivées partielles, Paris (1962) (Paris Editions du C.N.R.S., ed.), pp. 87-89 (1963)

[15] Matano, H.: Convergence of solutions of one-dimensional semilinear heat equations. J. Math. Kyoto Univ. 18, 221-227 (1978)

[16] Simon, L.: Asymptotics for a class of non-linear evolution equations, with applications to geometric problems. Ann. Math. 118, 525-571 (1983)

[17] Zelenyak, T.I.: Stabilization of solutions of boundary value problems for a second-order parabolic equation with one space variable. Differ. Equ. 4, 17-22 (1968) (Transl. from Differ. Uravn. 4 (1968) 34-45)

Daniel Hauer

Institut für Angewandte Analysis

Universität Ulm

89069 Ulm

Germany

e-mail: daniel.hauer@uni-ulm.de 
Daniel Hauer

Laboratoire de Mathématiques et Applications de Metz Université de Lorraine et CNRS

UMR 7122

Bât. A Ile du Saulcy 57045 Metz Cedex 1

France

e-mail: hauer@math.cnrs.fr

Received: 5 April 2012.

Accepted: 8 October 2012. 